\title{
Mecanobiología de los huesos maxilares. II. Remodelación ósea
}

\author{
Cano-Sánchez J*, Campo-Trapero J**, Sánchez-Gutiérrez JJ***, \\ Bascones-Martínez A****
}

\section{RESUMEN}

La mecanobiología ósea se encarga de la interacción entre las señales mecánicas y los mecanismos moleculares en las células del tejido óseo. El proceso de remodelado óseo se ve influenciado por las cargas biomecánicas a diferentes niveles estructurales. En este artículo se revisa la relación entre la carga y la expresión molecular durante el remodelado óseo e intenta describir las diversas teorías que explican la transducción de señales de carga que parecen influir en la transmisión de cargas perimplantarias.

Palabras clave: Mecanobiología, hueso, implantes.

\section{SUMMARY}

Bone mechanobiology deals with connection between mechanical signals and molecular events in cells and bone tissue. Remodelling process can be influenced by biomechanical loading in different structural levels. This review paper tries to show the connection between loading and molecular expresion during bone remodelling and describes some theories which deals with signals of transduction which seem to have some importance in perimplantary loading transmision.

Key words: Mechanobiology, bone, implants.

Fecha de recepción: Septiembre 2007.

Aceptado para publicación: Septiembre 2007.

* Profesor Asociado. Facultad de Odontología. UCM.

** Profesor Contratado Doctor. Facultad de Odontología. UCM.

*** Medico adjunto del Servicio de Cirugía Oral y Maxilofacial. Hospital Clínico San Carlos. Madrid.

**** Catedrático. Facultad de Odontología. UCM.

Cano-Sánchez J, Campo-Trapero J, Sánchez-Gutiérrez JJ, Bascones-Martínez A. Mecanobiología de los huesos maxilares. II. Remodelación ósea. Av. Odontoestomatol 2008; 24 (2): 177-186.

\section{INTRODUCCIÓN}

Las cargas biomecánicas tienen una influencia en la biología ósea tanto en el proceso de regeneración ósea (formación de nuevo hueso tras un daño en el mismo) como en el del remodelado óseo (forma- ción de nuevo hueso para cambiar el hueso antiguo o madurar el hueso regenerado). De hecho, ambos procesos se solapan en su fisiología de tal forma que cuando se origina un proceso de regeneración ósea se produce también un fenómeno de remodelado en los bordes óseos que han sufrido el trauma con el fin 
de eliminar el hueso necrótico. Por otro lado, cuando se produce la activación de una unidad de remodelado óseo (osteona o trabécula) se origina también un proceso de regeneración ya que se activa toda la maquinaria celular y molecular para la neoformación ósea.

Con el fin de sistematizar, en este trabajo se describen los eventos biomecánicos que influyen en el remodelado óseo, y los que influyen en la regeneración ósea se presentarán en una publicación posterior. El presente artículo intenta establecer la relación entre la carga y la expresión molecular durante el remodelado óseo y describir las diversas teorías que explican la transducción de señales de carga que parecen influir en la transmisión de cargas perimplantarias.

\section{INFLUENCIA DE LA CARGA EN LA EXPRESIÓN MOLECULAR DURANTE EL REMODELADO ÓSEO}

Los osteocitos son células con una morfología aplanada y alargada, de aproximadamente $10 \mu \mathrm{m}$ de longitud mayor, con numerosas prolongaciones (procesos celulares) que se introducen en los canalículos óseos (canalículos de 10-100 nm de diámetro). Se ha sugerido que son células capaces de responder a estímulos antagónicos de carga activando osteoclastos u osteoblastos, en función de la carga local. Aunque el proceso de comunicación es motivo de investigación, se están describiendo una serie de moléculas implicadas en el fenómeno de transducción de cargas (1).

Para la traducción de los estímulos mecánicos externos (fluido canalicular) en señales intracelulares dentro del osteocito, se hacen indispensables diversos receptores de membrana, como son las integrinas y los receptores CD44. Posteriormente, el osteocito manda sus señales externamente mediante diferentes mediadores, por ejemplo $\mathrm{PGE}_{2}$, que a su vez induce una subsiguiente producción de cicloxigenasa2 (COX-2), la que parece que facilita la producción de prostaglandinas incluso varias horas después que se haya eliminado la carga (memorización de los eventos de estrés) (2).

La prostaglandina E2 $\left(\mathrm{PGE}_{2}\right)$ es producida principalmente por los osteocitos y también por otras cé- lulas del linaje osteoblástico en respuesta a una carga mecánica representada por el flujo del fluido canalicular. Se ha visto que esa producción continúa incluso 24 horas después del cese del estimulo mecánico (3). La PGE 2 es constituida por la acción de la enzima ciclooxigenasa-2 (COX-2) que presenta un rápido incremento tras la aplicación de carga sobre las células óseas. Se ha observado in vitro que la COX-1 no tiene influencia en la producción de $\mathrm{PGE}_{2}$ en las células óseas por estímulo mecánico.

La PGE 2 tiene tanto efectos anabólicos como catabólicos, es decir tiene la capacidad de estimular la formación de osteoclastos, a través de la producción de CSF (por Colony Stimulating Factor) y el RANKL (por Receptor Activator of Nuclear factor Kappa-b Ligand) en los osteoblastos (4), y también tiene la capacidad de estimular o inhibir el crecimiento y la diferenciación de osteoblastos según su concentración (5). El RANKL se considera esencial para la diferenciación de osteoclastos. Es producido por los osteoblastos y por algunos precursores osteoclásticos. Se ha relacionado también con la inducción de osteopontina (OPN) dentro de los osteoclastos. Su receptor en los preosteoclastos es bloqueado por la osteoprotegerina (OPG) (6).

Se ha demostrado con modelos animales que la aplicación continua de la carga crea una saturación en la respuesta de las células osteogénicas (7). Sin embargo la presencia de pequeños periodos de descanso (10-15 segundos) entre los periodos de carga, permite el restablecimiento de la sensibilidad celular al estímulo mecánico (8).

El osteocito también manda oxido nítrico (NO) al exterior como respuesta a la adecuada deformación de su membrana, produciendo una disminución de la apoptosis de los mismos y una inhibición subsiguiente de los osteoclastos (1). Se ha observado in vitro con células de médula ósea que el NO reduce la expresión de RANKL y aumenta la expresión de OPG por parte de los preosteoblastos y osteoblastos, lo que inhibe el reclutamiento de células mononucleares para formar osteoclastos (9). De manera paradójica, se ha observado que una baja producción de NO inhibe la apoptosis celular mediante la inhibición de la caspasa 3, la producción de proteínas de choque calórico y la estimulación de Bcl-2. 
Pero las altas producciones de NO también promoverían la apoptosis. Lo que parece indicar que la producción de NO tendría efectos controvertidos en la activación de la apoptosis osteocitaria (10).

Mediante la determinación de fosfatidilserina (marcador de apoptosis) en cultivos de osteocitos, se ha observado que el flujo de fluido canalicular fisiológico $(0,6 \pm 0,3 \mathrm{~Pa}$ a una frecuencia de $5 \mathrm{~Hz})$ inhibe la apoptosis de los mismos, mientras que el estancamiento del fluido la favorece. Este proceso de apoptosis parece mediado por Bcl-2 (proteína antiapoptótica) que se ve aumentada cuando se aplica un estrés de cizallamiento sobre los osteocitos. Se ha observado que este fenómeno no afecta a los osteoblastos de superficie (lining cells), siendo específico de los osteocitos. Esto confirma la hipótesis de que el estrés del fluido interviene sobre el sistema canalicular pero no en las superficies del mismo (11).

Se ha demostrado la correlación entre la osteopontina (OPN) y la transducción intracelular de señales mecánicas con un consiguiente estimulo de la formación ósea $(12,13)$. La OPN puede ser expresada por fibroblastos, osteoblastos u osteoclastos, aunque el mayor productor de OPN son los osteocitos como principales protagonistas de la mecano-transducción. La OPN se une a la integrina $\alpha_{v} \beta_{3}$ de los preosteoclastos siendo importante en el reclutamiento y diferenciación de los preosteoclastos en la zona de carga ósea, mediante la adhesión a la matriz ósea $(12 ; 14)$. Se ha observado también una mayor expresión de OPN en las zonas de producción de hueso inmaduro por parte de los osteoblastos, por lo que se sugiere que la OPN tiene una doble función tanto en la reabsorción como en la formación del remodelado inducido por carga (12).

En un modelo experimental (6) de sobrecarga en dientes naturales se ha observado la expresión aumentada de OPN y RANKL en el ligamento periodontal. La expresión de OPN se observó en las zonas de compresión en los osteoblastos y fibroblastos. Pero también se observó una mayor producción de OPN por parte de los osteoclastos lo cual se establece en la literatura como controvertido. Sin embargo, hay que tener en cuenta que el proceso molecular que se produce en el ligamento periodontal puede ser diferente al originado dentro de la matriz ósea, y quizá en otro ambiente los osteoclastos sean incapaces de expresar OPN.

Se ha demostrado que la aplicación de una carga estática o continua produce una expresión de OPN en los osteocitos $(12 ; 13)$, sin embargo la aplicación de una carga dinámica o intermitente (por ejemplo: carga oclusal) origina muy poca expresión de OPN en los osteocitos (6). Esto parece indicar que la producción de OPN y su función de activación de los osteoclastos tienen diferentes vías según el tipo de carga aplicada (6). La producción de OPN también se ve influida por la edad, de tal manera que su expresión se ve reducida con la misma (15).

De reciente interés, es el estudio de la proteína Wnt y sus cascadas moleculares intracelulares. El nombre proviene de la unión del gen de drosophila "Wingless" y el del ratón "int". Es una proteína de señal extracelular expresada por diferentes tipos de células. Existen 12 tipos de proteínas. Presenta un receptor con siete dominios transmembrana (Frizzled) que tiene como correceptor el LRP5 (por low-density Liprotein Receptor-related Protein 5). Las proteínas intracelulares de esta cascada incluyen Dsh (Dishevelled), Axin, GSK3 (por Glycogen Synthase Kinase), $\beta$-catenina (armadillo). Cuando no hay señal de Wnt la GSK3 fosforila $\beta$-catenina por lo que se ubiquitiniza y es destruida por el proteosoma. Cuando Wnt se une a su receptor, se activa Dsh, que inhibe la GSK3, con lo que se libera axin de $\beta$-catenina que se acumula en el citoplasma y pasa al núcleo donde se une al factor de transcripción TCF para expresar diferentes genes implicados en la biología ósea (16). Hay varias proteínas extracelulares que pueden inhibir este proceso, por ejemplo: Dkk (Dickkopf), Kremen o esclerostina se une a LRP5, impidiendo la unión a Frizzled y por tanto evitando la internalización de la señal de Wnt.

La señal de Wnt presenta expresión de 879 genes (17), que tienen como función, entre otras, el control de la diferenciación osteoblástica, inhibición de la apoptosis y producción de proteínas de la vía Wnt (retroalimentación). De particular interés es la sobrerregulación de OPG, proteína que disminuye la osteoclastogenesis al bloquear el RANKL. 
La esclerostina es una proteína extracelular que se une al receptor LRP5, inhibiendo la vía Wnt. Es expresada únicamente en el hueso por los osteocitos de la profundidad del hueso, pero no por los osteocitos de las superficies óseas. Una teoría establece que la esclerostina inhibiría los osteocitos de superficie (lining cells) y cuando se necesita la formación de nuevo hueso se eliminaría esa inhibición producida por la esclerostina. Cuando los osteocitos internos son dañados por una microfractura, y entran en apoptosis, dejarían de mandar la "señal inhibitoria" de esclerostina, con lo que esas células permiten el reclutamiento de preosteoblastos, diferenciación de osteoblastos y formación de hueso. La esclerostina favorecería la osteoclastogénesis por la inhibición selectiva en el hueso de la vía Wnt, por lo que se esta estudiando su inhibición como terapia selectiva en la osteoporosis (16).

\section{TEORÍAS SOBRE EL MECANISMO CELULAR Y MOLECULAR DEL REMODELADO ÓSEO SOMETIDO A CARGA}

A pesar de los avances en el conocimiento de los procesos de remodelado óseo, muchos de los acontecimientos que relacionan ese remodelado con la distribución de las cargas permanece desconocido. Durante el remodelado, el alineamiento del nuevo hueso se va formando a lo largo de la dirección de la carga local dominante, lo que sugiere una regulación de la formación ósea por parte de los estímulos mecánicos. Durante décadas ha quedado demostrado que los osteocitos son los mecanosensores del hueso, y que la porosidad canalículo-lacunar es la estructura encargada de mediar la mecanosensibilidad (1). También se ha evidenciado que la carga mecánica induce el movimiento del fluido canalicular en el interior de la estructura, y a partir de ahí, se ha sugerido que ese movimiento sería un mediador de la mecanosensibilidad de los osteocitos. Se ha sugerido también que los osteocitos responderían produciendo moléculas que modularían la actividad de osteoblastos y osteoclastos, y de esta manera convertir la señal mecánica en una señal celular (18).

Las cargas aplicadas sobre el hueso se suelen establecer en medidas de deformación en $\mu \varepsilon$ (microstrain), que es un ratio entre la longitud de un objeto someti- do a estrés, y la longitud original del objeto. En esta escala matemática, $10^{6} \mu \varepsilon$ implicaría una teórica deformación del 100\% (10.000 $\mu \varepsilon$ es un $1 \%$ de deformación) (19). Según Frost el hueso tendría un control (similar a un termostato) mediante el modelado y el remodelado, que lo permitiría acoplarse a las condiciones de cargas que recibe del exterior ("teoría del mecanostato"). Según esta adaptación en las BMU, la diferencia entre la cantidad de hueso reabsorbido y el hueso depositado vendría expresado por la "fracción rho" $(\rho)(20)$. Un $\rho$ positivo indica un incremento de la deposición ósea mientras que un $\rho$ negativo un incremento de la reabsorción. Frost estableció 4 niveles de adaptación del hueso a las cargas, en las llamadas "ventanas de uso mecánico" (mechanical usage windows).

Basado en las categorías de Frost (20), Wiskott estableció 5 categorías (una más que las indicadas por Frost y con diferentes rangos), mediante estimaciones teóricas de la influencia de las cargas en el hueso, ya que los rangos exactos son difíciles de aplicar por influencia de diferentes parámetros (densidad ósea, frecuencia de carga, historial de las cargas aplicadas, gradientes de carga, repercusión de factores locales u hormonales...) (19):

- Desuso (mayor reabsorción ósea): Se produce con unas cargas menores a $100 \mu \varepsilon$. El índice $\rho$ es negativo por una mayor fase de reabsorción en los ciclos de remodelado.

- Carga fisiológica normal (homeostasis ósea): En el rango 100-1.500. La fracción $\rho$ es cero.

- Sobrecarga media (mayor aposición ósea): En el rango $1.500-4.000 \mu \varepsilon$. El $\rho$ es positivo.

- Sobrecarga patológica (Daño óseo irreversible): Por encima de $4.000 \mu \varepsilon$ (0,4\% de deformación). Aparecen fenómenos de movilidad y grietas por fatiga. Aunque no está del todo aclarado, se ha establecido en estudios de elementos finitos, que se produce un aumento de la remodelación con mayor fase reabsortiva, apareciendo un $\rho$ negativo. En el hueso perimplantario este fenómeno se traduciría en una reabsorción del hueso en contacto con el implante, con movilidad del implante que previamente estaba osteointegrado.

- Fractura: La resistencia a la flexión del hueso es aproximadamente de 120 Mpa, lo que se corresponde a una deformación de $20.000 \mu \varepsilon$ ( $2 \%$ de deformación). 
Se ha establecido más definitivamente que tanto el desuso como la sobrecarga media ósea aumentarían la remodelación, en un caso con un aumento de la reabsorción y en el otro de aposición.

Se ha demostrado que durante la formación ósea ciertos osteoblastos escapan de la muerte por apoptosis y aproximadamente el $15 \%$ en el hueso cortical y el $30 \%$ en el hueso trabecular se convierten en osteocitos. El mecanismo por el cual unos osteoblastos son seleccionados para ser osteocitos y otros no, es desconocido (21).

Durante ese remodelado los osteoclastos aparecen primero cavando un "túnel" a través del hueso compacto o una "trinchera" en la superficie del hueso trabecular. Ese espacio es rellenado por nuevo hueso a través de la actividad de los osteoblastos. El gran misterio reside en como los osteoclastos y osteoblastos pueden acoplar su actividad para actuar en la misma dirección en la que se está aplicando la carga dominante sobre un determinado hueso. Existen varias teorías que explican el acoplamiento de ambos tipos celulares. Algunas de ellas basan el acoplamiento osteoblastos-osteoclastos en factores no mecánicos. De esta manera Rodan y Martin (22) establecieron que los osteoblastos son los encargados de indicar a los osteoclastos cuando y donde reabsorber la matriz extracelular, basándose en que los osteoblastos presentan receptores hormonales y posteriormente emiten señales moleculares que regulan la actividad osteoclástica. Por tanto la influencia hormonal de los osteoclastos estaría mediada por los osteoblastos. Otra teoría establece que ese "acoplamiento" entre osteoblastos y osteoclastos reside en que cuando se reabsorbe la matriz extracelular se liberan factores de crecimiento que estimulan a los osteoblastos a formar matriz (23).

Otras teorías han propuesto diferentes modelos para explicar el acoplamiento entre osteoclastos y osteoblastos, pero en este caso teniendo más presente las cargas biomecánicas.

Hoy en día se ha aceptado que la reacción de las células óseas a las cargas biomecánicas viene regulado por el flujo pulsátil del fluido canalicular (18). En principio se pensó que la transmisión de señales dependía de diferencias de potencial según avanza- ba el fluido y también se indicó la presencia de factores nutricionales como mensajeros de la respuesta mecánica. Sin embargo, hoy en día se asume que la respuesta celular depende de los factores mecánicos que aporta el fluido sobre los osteocitos.

Se ha descrito con células in vitro que la fuerza de cizallamiento que ejerce el fluido sobre la membrana del osteocito produce emisión de señales bioquímicas (18), sin embargo este experimento no tiene en cuenta las fibras de "amarre" exteriores al osteocito que impedirían su cizallamiento libremente. Parfitt (21) se decanta más por el arrastramiento del fluido de las fibras de amarre entre el osteocito y las paredes óseas de la laguna o el canalículo. Demostrado in vitro, esta deformación de las fibras de amarre produce una deformación "en aro" o circular a lo largo de la membrana del osteocito que induciría la respuesta bioquímica (24).

De esta manera, cualquiera que sea el modo por el que el osteocito detecta la deformación (deformación por cizallamiento o deformación circular), cuando esa deformación es muy alta, se activa el proceso para formar hueso en las superficies endósticas y periósticas. Pero cuando la deformación es muy baja se reabsorbe hueso solo de las zonas endósticas, en un proceso de remodelación hasta alcanzar el rango habitual en el que el osteocito se encuentra cómodo. ¿Cuál es el proceso bioquímico que se genera tras la detección del cambio de deformación en el osteocito? y ¿cuál es el mecanismo que establece la dirección del acoplamiento entre osteoclastos-osteoblastos tras la alteración del fluido canalicular? Se han propuesto varias teorías para explicar estos fenómenos.

\section{Teoría de las microgrietas}

La carga cíclica sobre el hueso origina fatiga la cual se refleja en la aparición de microgrietas (microcraks). Estas microgrietas suele tener una longitud de 100 mm cuando son observadas en una sección, y de mayor longitud cuando son observadas en tres dimensiones. Estas grietas causan un gran aumento local de la deformación a la que se ve sometido el osteocito. Esa deformación es transmitida (se desconoce si es un proceso bioquímico en el interior de los osteocitos o bien mecánico del fluido lacuno- 
canalicular) hasta las células de revestimiento endostales. Estas células se activan, cambian su forma para retirar la membrana endostal y envían señales (producción de RANKL en su membrana) para atraer a las células mononucleares preosteoclásticas provenientes de los vasos. De esta forma se crearía una nueva BMU que retira el hueso que incluye la microgrieta y regenera uno nuevo (21).

La presencia de microdefectos en el hueso tiene repercusiones tanto en la fisiología del hueso como en sus propiedades biomecánicas. Por un lado intervienen en la fisiología del sistema de malla osteocitario y por otro lado reducen los valores de rigidez, resistencia y dureza del hueso (25).

El acúmulo de daño por fatiga se va incorporando en los huesos en forma de microdefectos. Se ha especulado sobre cual es la causa de que existan más microdefectos con la edad: a) como resultado de una historia de acúmulo de microdefectos; b) existiría alguna alteración de la matriz a nivel de la micro o nanoescala; c) existiría alguna alteración para la reparación de los defectos cuando se incrementa la edad. Se ha afirmado que las microgrietas que aparecen en el hueso son consecuencia de la presencia de un hueso muy antiguo en la matriz intersticial que no ha sido remodelado adecuadamente y por tanto tiene una mayor mineralización y fragilidad. Con estudios mecánicos a macroescala se ha observado que un contenido mineral mayor del $70-75 \%$ del peso total del hueso sería crítico para la fragilidad de la estructura (26). Sin embargo, utilizando sistemas de nanoindentación se ha observado que el hueso que alberga las microgrietas tiene características similares de elasticidad, índice de deformación y plasticidad a las del resto del hueso, lo que cuestionaría el contenido mineral como único justificante de los microdefectos. Parece que alteraciones en la dureza debido a alteraciones en la estructura del colágeno en hueso antiguo podría tener una mayor repercusión en la génesis de las microgrietas (27).

\section{Teoría del NO}

Burger y cols (28) establecen que la orientación del remodelado y el diámetro de la osteona o hemi-osteona es establecido por los osteoclastos. Se des- conoce sin embargo el mecanismo por el cual esos osteoclastos son dirigidos en su actividad hacia una determinada dirección marcada por la tensión establecida. Martin propuso en 1982 (29) que los osteoclastos son activados por el estrés tensional, mientras que los osteoblastos son activados por el estrés compresional. Sin embargo, se ha evidenciado que en ocasiones esa relación no se corresponde siempre así, por lo que la existencia local de tensión o compresión no puede explicar por si sola el comportamiento de osteoclastos y osteoblastos (30).

Varios estudios han evidenciado la tarea como mecanosensores que tienen los osteocitos en la matriz ósea mineralizada (31). Teniendo presente la evidencia de la transducción de señales a través de fluido canalicular, Burger y cols (28) realizaron un estudio mediante elementos finitos en el que observaron diferencias en el fluido canalicular en la punta y la base de un cono de corte de remodelado sometido a carga, indicando que los osteocitos de estas zonas recibían diferentes informaciones mecánicas.

Se ha visto que los osteocitos, a través de la enzima NO sintasa (ecNOS, por endotelial cell Nitric Oxyde Synthase) producen oxido nítrico a partir de L-arginina, cuando detecta un estrés de cizallamiento en el fluido canalicular, de manera similar a lo que les ocurre a las células endoteliales como respuesta al fluido de la sangre (32). En las células endoteliales la producción de $\mathrm{NO}$ en respuesta al fluido juega un papel importante para evitar la apoptosis de las células endoteliales, ya que el NO rechaza los monolitos (células inflamatorias) de las superficie endotelial. Observando este modelo en las células endoteliales, Burger y cols (28) mediante modelos de elementos finitos han establecido la hipótesis de que el oxido nítrico actúe de manera similar en el hueso, de tal manera que en la punta del cono de corte debido al insuficiente fluido canalicular (éxtasis del fluido con menor deformación sobre los osteocitos), los osteocitos no producirían NO y entrarían en apoptosis. Al igual que en las células endoteliales, la apoptosis de los osteocitos atraerían a los macrófagos que reabsorberían la matriz extracelular para fagocitar los osteocitos muertos. De manera similar a los macrófagos, los osteoclastos serían atraídos por las células apoptóticas que expresan fosfatidilserina en su membrana. En la base del cono de corte (cono de cierre- 
zona de inversión y formación del cono), debido a que los osteocitos serían bien estimulados por un fluido canalicular adecuado, se produciría NO, no se produciría la apoptosis de esos osteocitos y los osteoclastos se separarían de la superficie de la matriz ya que en esa zona no tienen nada que fagocitar.

Esta teoría estaría avalada por la evidencia de que en hueso sano el diámetro del cono de corte de las osteonas secundarias permanece bastante similar y constante. Indicando que los osteoclastos siempre dejan de actuar cuando se alcanza un diámetro determinado que permite un fluido canalicular adecuado que regula la producción de NO por los osteocitos. Esta teoría no explicaría el arranque del proceso de remodelado (mejor representado por la teoría de las microgrietas o teorías endocrinas), pero si explicaría el avance del cono o hemicono de corte siguiendo la dirección de la carga.

\section{Teoría de la señal inhibitoria de los osteocitos}

Martin (33) estableció la hipótesis que los osteocitos, como mecanosensores, mantendrían continuamente una señal inhibitoria (de naturaleza molecular desconocida) con las células de revestimiento para que no se inicie la remodelación. Parece que las cargas normales mantendrían esa señal inhibitoria, y al tener un desuso importante desaparecería esa señal favoreciendo la remodelación. Por otro lado en casos de sobrecarga moderada existirían microfracturas que podrían inducir la apoptosis de los osteocitos, o eliminar la comunicación osteocito-célula de revestimiento, que también rompería la señal inhibitoria. Hay algunos autores que establecen que esa señal inhibitoria podría estar determinada por esclerostina (16).

Otro mecanismo de activación local de la remodelación se produce cuando hay una necrosis de los osteocitos (por ejemplo: en traumatismo o en la colocación de un implante) en este caso los osteocitos dejarían de mandar a las célula de revestimiento la señal de inhibición que impiden el remodelado, y por lo tanto activándolo (33). También la reducción de estrógenos (menopausia) induciría la apoptosis de los osteocitos aumentando la remodelación y haciendo que la fase de reabsorción sea mas grande en extensión que en condiciones normales. Parece además, que la comunicación de esas células de revestimiento al "sincitio osteocitario", evitarían que esos osteoblastos desaparezcan por apoptosis una vez que terminan de formar hueso.

Esta teoría inhibitoria de la comunicación osteocitocélula de revestimiento, podría ser también aplicada a el proceso de modelado. Sin embargo la misma señal que puede aumentar el remodelado, puede disminuir el modelado en función de la localización anatómica o ultraestructural (por ejemplo: acción diferente en periostio o endostio). Así en localizaciones periósticas, las modificaciones de esa señal podría generar un proceso de modelado en vez de remodelado, de tal forma que las células de revestimiento recibirían una señal que las induciría directamente el fenotipo osteoblástico (modelado anabólico) o a producir moléculas que diferencien osteoclastos (modelado catabólico). Por ejemplo: un aumento moderado de la carga biomecánica detectada por los osteocitos cercanos a una localización perióstica, produciría una perdida de la señal inhibitoria entre osteocitos-células de revestimiento que en esta localización no va a producir el inicio de la remodelación sino un proceso directo de diferenciación fenotípica osteoblástica, que originaría un proceso de modelado anabólico en esa zona. Por otro lado una falta de cargas provocaría un efecto de modelado catabólico directo con reabsorción ósea (33).

Se ha evidenciado en varios trabajos que la muerte de los osteocitos (por necrosis o apoptosis) favorece la activación del remodelado mediante la creación de nuevas BMU (34). Sin embargo, es desconocido si esa activación del remodelado se produce por la pérdida de una señal que es normalmente inhibitoria sobre las células de revestimiento (como indica Martin, 2000) o bien por la transmisión a las células de revestimiento de una señal positiva o activadora cuando mueren los osteocitos (33).

\section{Teoría del intercambio de iones calcio}

Turner y Robling (35) también establecen una teoría sobre posible mecanismos molecular que rigen la mecanotransducción y la activación del recambio óseo. Según los autores, el flujo del fluido produciría 
un estrés de cizallamiento sobre los osteocitos que generaría una entrada de iones $\mathrm{Ca}^{2+}$ a través de canales iónicos y otros supuestos canales de mecanostransducción. Esta entrada produciría un aumento del $\mathrm{Ca}^{2+}$ citosólico lo que activa la liberación de ATP. Este ATP expulsado se uniría a determinados receptores acoplados a proteína G (GPCR, por GProtein Coupled Receptor) (receptor $\mathrm{PRY}_{2}$ y $\mathrm{P}_{2} \mathrm{X}_{7}$ ). La unión de ATP a estos receptores activaría la fosfolipasa C (PLC, por Phospholipase C) que actúa sobre el fosfatidilinositol difosfato ( $\mathrm{PIP}_{2}$, por phospatidylinositol biphospate) dividiéndolo en Inositol trifosfato ( $\mathrm{IP}_{3,}$ por inositol triphosphate) y diacilglicerol. $\mathrm{IP}_{3}$ se transloca al retículo endoplásmico donde se une a receptores de $\mathrm{Ca}^{2+}$ y así favorece su liberación al citoplasma. La concentración de $\mathrm{Ca}^{2+}$ citoplasmática además de liberar ATP extracelular y distribuir la señal, parece que podría mediar la liberación por parte de los osteocitos de $\mathrm{PGE}_{2}$. Estas prostaglandinas activarían los receptores $\mathrm{EP}_{2}$ y $\mathrm{EP}_{4}$ en los osteoblastos y células de revestimiento activando vías intracelulares de formación de matriz ósea. También se sugiere que los receptores de PTH estarían mediando en la transmisión de la señal mecánica, pero su mecanismo es desconocido.

De manera general se puede establecer con una aceptación mayoritaria cual es el camino de la mecanotransducción del hueso: Carga en el hueso $\rightarrow$ deformación de la matriz extracelular $\rightarrow$ Flujo del fluido canalicular $\rightarrow$ activación de los osteocitos (células sensores) $\rightarrow$ producción de mensajeros quími$\cos \rightarrow$ respuesta metabólica de osteoblastos/osteoclastos (células efectoras).

\section{BIBLIOGRAFÍA}

1. Klein-Nuleng J, Bacabac RG, Mullender MG. Mechanobiology of bone tissue. Path Biologie 2005;53:576-80.

2. Sterck JGH, Klein-Nulend J, Lips P, Burger EH. Response of normal and osteoporotic human bone cells to mechanical stress in Vitro. Am J Physiol 1998;274:1113-20.

3. Bakker AD, Klein-Nulend J, Burger EH. Mechanotransduction in bone cells proceeds via activation of COX-2, but not COX-1. Biochem Biophys Res Comm 2003; 305: 677-683.

4. Suzawa, T, Miyaura, C, Inada, M, Maruyama, T, Sugimoto, Y, Ushikubo, F, Ichikawa, A, Narumiya, $\mathrm{S}$, Suda, T. The role of prostaglandin $\mathrm{E}$ receptor subtypes (EP1, EP2, EP3, and EP4) in bone resorption: an analysis using specific agonists for the respective EPs. Endocrinology 2000;141, 1554-9.

5. Raisz LG, Fall PM. Biphasic effects of Prostaglandin E2 on bone formation in cultured fetal ral calvariae: interacttions with cortisol. Endocrinology 1990;126 (3):1654-9.

6. Kaku M, Uoshima K, Yamashita Y, Miura H. Investigation of periodontal ligament reaction upon excessive occlusal load. Osteopontin induction among periodontal ligament cells. J Periodont 2005;40:59-66.

7. Turner $\mathrm{CH}$. Three rules for bone adaptation to mechanical stimuli. Bone 1998; 23 (5), 399-407.

8. Robling AG. Burr DB. Recovery periods restore mechanosensitivity to dynamically loaded bone. Journal of Experimental Biology 2001;204:3389-99.

9. Fan X, Roy E, Zhu L, Murphy TC, Ackert-Bicknell C, Hart CM, Rosen C, Nanes MS, Rubin J. Nitric oxide regulates RANKL and OPG expression in bone marrow stromal cells. Endocrinology 2004;14582):751-9.

10. Dimmeler S, Zeiher AM. Nitric oxide and apoptosis: another paradigm for the double-edged role of nitric oxide. Nitric Oxide 1997;1(4):275-81.

11. Bakker A, Klein-Nulend J, Burger E. Shear stress inhibits while disuse promotes osteocyte apoptosis. Biochem Biophysic Res Com 2004;320: 1163-8.

12. Terai K, Takano-Yamamoto T, Ohba Y, Hiura K, Sugimoto M, Sato M, Kawahata H, Inaguma N, Kitamura Y, Nomura S. Role of osteopontin in bone remodeling caused by mechanical stress. J Bone Miner Res 1999;14(6): 839-49. 
13. Morinobu M, Ishijima M, Rittling SR, Tsuji K, Yamamoto H, Nifuji A, Denhardt DT, Noda M. Osteopontin expression in osteoblasts and osteocytes during bone formation under mechanical estress in the calvarial suture in vivo. J Bone Miner Res 2003;18(9):1706-15.

14. Miyauchi A, Alvarez J, Greenfield EM, Teti A, Grano M, Colucci S,Zambonin-Zallone A, Ross FP, Teitelbaum SL, Cheresh D Recognition of osteopontin and related peptides by an alpha v beta 3 integrin stimulates immediate cell signals in osteoclasts. J Biol Chem 1991;266(30):20369-74.

15. Ikeda T, Nagai Y, Yamaguchi A, Yokose S, Yoshiki $\mathrm{S}$. Age related reduction in bone matrix protein mRNA expression in rat bone tissues: application of histomorphometry to in situ hybridization. Bone 1995;16:17-23.

16. Ott, S. Sclerostin and Wnt signalling- The pathway to bone strenght. J Clin End Metab 2005; 90(12):6741-3.

17. Jackson A, Vayssiere B, García T, Newell W, Baron R, Roman-Roman S, Rawadi G. gene array analysis of wnt-regulated genes in C3H10T1/2 cells; Bone 2005;36:585-98.

18. Weinbaum S, Cowin Sc, Zeng Y. A model for the excitation of osteocytes by mechanical loadinginduced bone fluid shear stresses. J Biomech 1994;27:339-60.

19. Wiskott HWA, Belser UC. Lack of integration of smooth titanium surfaces: a working hypothesis based on strains generated in the surrounding bone. Clin Oral Impl Res 1999;10:429-44.

20. Frost HM. Wolff's law and bone's structural adaptations to mechanical usage: an overview for clinicians. The Angle Orthodontics 1994;64: 175-88.

21. Parfitt AM. "Osteocytes: The link between strain, structure and strenght". En: "ASBMR. 26 $6^{\text {th }}$ Annual Meeting Webcast. Plenary Symposium II: New insights into bone strenght: Harold M. Frost memorial session". http://app2.capitalreach.com/ esp $1204 /$ servlet $/ \mathrm{tc} ? \mathrm{cn}=\mathrm{asbmrEc}=10169 \& \mathrm{~s}=$ 20282\&e $=3345 \varepsilon \varepsilon .2004$.

22. Rodan GA, Martin TJ. Role of osteoblasts in hormonal control of bone resorption-a hypothesis. Calcified Tissue Internacional 1981;33:349-51.

23. Parfitt AM. Mundy GR, Roodman GD, Hughes $\mathrm{DE}$, Boyce BF. A new model for the regulation of bone resorption, with particular reference tp the effects of bisphosphonates. Journal of Bone and Mineral Research 1996;11:150-9.

24. You L, Cowin SC, Schaffler MB, Weinbaum S. Amodel for strain amplification in the actin cytoskeleton of osteocytes due to fluid drag on pericellular matrix. J Biomech 2001;34:1375-86.

25. Zioupos P. Accumulation of in vivo fatigue microdamage and its relation to biomechanical properties in ageing human cortical bone. J Microsc 2001;201:270-8.

26. Currey JD. Mechanical properties of bone with greatly differing functions. J Biomech 1979;12:313-9.

27. Zioupos P. In vivo fatigue microcracks in human bone. Material properties of the surrounding bone matrix. Eur J Morphol 2005;42:3-41.

28. Burger EH, Klein-Nulend J, Smit TH. Strain derived canlicular fluid flow regulates osteoclast activity in a remodelling osteon. A proposal. J Biomechanics 2003;36:1453-9.

29. Martin RB, Burr DB. A hypothetical mechanism for the stimulation of osteonal remodelling by fatigue damage. J Biomech 1982;15(39):137-9.

30. Smit TH. Burger EH. Is BMU-coupling a strainregulated phenomenon? A finite element analysis. J Bone Min Res 2000;15:301-7.

31. Burger EH, Klein-Nulend J. Mechanotransduction in bone-role of the lacuno-canalicular network. FASEB Journal 1999;13:S101-S112.

32. Uematsu M, Navas JP, Nishida K, Ohara Y, Murphy TJ, Alexander RW, Nerem RM, Harrison DG. 
Regulation of endothelial cell nitric oxide synthase mRNA expression by shear stress. American Journal of Physiology 1995;269: 1371-8.

33. Martin RB. Toward a unifying theory of bone remodeling. Bone 2000;26(1):1-6.

34. Verborgt O, Gibson GJ, Schaffler MB. Loss of osteocyte in association with microdamage and bone remodeling after fatigue in vivo. $\mathrm{J}$ Bone Miner Res 2000;15(1):60-7.
35. Turner $\mathrm{CH}$, Robling GA. Exercise as an anabolic stimulus for bone. Curr Pharmac Des 2004; 10(21):2629-41.

\section{CORRESPONDENCIA}

Jorge Cano Sánchez Carballino, 21, $1^{\text {a }}$ dcha. 28925 Alcorcón (Madrid) email: jo.cano@wanadoo.es 